\title{
Estimating trace deposition time with circadian biomarkers: a prospective and versatile tool for crime scene reconstruction
}

\author{
Katrin Ackermann • Kaye N. Ballantyne • \\ Manfred Kayser
}

Received: 8 February 2010 /Accepted: 31 March 2010 /Published online: 24 April 2010

(C) The Author(s) 2010. This article is published with open access at Springerlink.com

\begin{abstract}
Linking biological samples found at a crime scene with the actual crime event represents the most important aspect of forensic investigation, together with the identification of the sample donor. While DNA profiling is well established for donor identification, no reliable methods exist for timing forensic samples. Here, we provide for the first time a biochemical approach for determining deposition time of human traces. Using commercial enzyme-linked immunosorbent assays we showed that the characteristic 24-h profiles of two circadian hormones, melatonin (concentration peak at late night) and cortisol (peak in the morning) can be reproduced from small samples of whole blood and saliva. We further demonstrated by analyzing small stains dried and stored up to 4 weeks the in vitro stability of melatonin, whereas for cortisol a statistically significant decay with storage time was observed, although the hormone was still reliably detectable in 4-week-old samples. Finally, we showed that the total protein concentration, also assessed using a commercial assay, can be used for normalization of hormone signals in blood, but less so in saliva. Our data thus demonstrate that estimating normalized concentrations of melatonin and cortisol represents a prospective approach
\end{abstract}

Electronic supplementary material The online version of this article (doi:10.1007/s00414-010-0457-1) contains supplementary material, which is available to authorized users.

K. Ackermann · K. N. Ballantyne $\cdot$ M. Kayser $(\bowtie)$

Department of Forensic Molecular Biology, Erasmus University

Medical Center Rotterdam,

PO Box 2040, 3000 CA Rotterdam, The Netherlands

e-mail: m.kayser@erasmusmc.nl

URL: www.erasmusmc.nl/fmb/ for determining deposition time of biological trace samples, at least from blood, with promising expectations for forensic applications. In the broader context, our study opens up a new field of circadian biomarkers for deposition timing of forensic traces; future studies using other circadian biomarkers may reveal if the time range offered by the two hormones studied here can be specified more exactly.

Keywords Melatonin - Cortisol · Biological rhythms . Circadian rhythm $\cdot$ Hormones $\cdot$ Forensic time estimation

$\begin{array}{ll}\text { Abbreviations } \\ \text { ELISA } & \text { Enzyme-linked immunosorbent assay } \\ \text { RIA } & \text { Radioimmunoassay } \\ \mathrm{T} & \text { Time point }\end{array}$

\section{Introduction}

Time estimation of a biological trace found at a crime scene represents one of the most important aspects in forensic casework, together with identification of the trace donor by DNA profiling. Knowledge about trace timing allows decisions to be made regarding whether the identified donor could potentially be the perpetrator and may also help to find potential eye witnesses or additional suspects. In principle, time aspects of crime scene samples can be seen from two sides. One side addresses the question of the time interval a sample was present at the crime scene, which may be addressable by differential degradation of biomarkers, such as different kinds of RNAs, but more detailed studies on this issue are needed [1]. The other side, 
which we address in this study, concentrates on determining the time point (i.e. the time of day) a trace was deposited at a crime scene, thereby bringing a chronobiological aspect into forensic research.

The 24-h day-night rhythm has always been the most reliable environmental cue for life on earth. Behavioural characteristics, metabolism and body functions are adjusted constantly to changing light and dark periods [2]. One of the best studied biomarkers of the human circadian pacemaker is the hormone melatonin, which signals to the body the duration of the dark period [3]. Melatonin is only produced and secreted during the night and shows its peak concentration in the later part of the night [3]. Cortisol is also widely used as a biological marker of circadian phase, often in parallel with melatonin [2, 4]. Cortisol levels display peak concentrations in the morning shortly after wakening and decrease throughout the day and night [5].

In this study, we investigated the feasibility of using circadian hormones, such as melatonin and cortisol, for estimating trace deposition time. We employed commercially available enzyme-linked immunosorbent assays (ELISA) easily applicable in forensic laboratories. To our knowledge, this is the first study exemplifying that deposition time of forensic traces can be estimated by means of circadian biomarkers, although the use of melatonin for time of death estimation has been described before $[6,7]$.

\section{Material and methods}

Sample collections and preparations

All samples investigated in this study were collected during an 8-week period in July to September 2009.

\section{Samples for ELISA applicability testing}

Three volunteers (two males, one female) under informed consent provided blood and saliva samples collected at the approximate peak and trough times of the two hormones of interest (cortisol: two males, 7-8 a.m. and 11-12 p.m.; melatonin: one male and one female, 3-4 a.m. and 3-4 p.m.). Two blood samples were collected per time point (one for serum preparation, one for sonication) by means of fingerpricks using contact-activated lancets (Becton Dickinson B.V., Breda, the Netherlands), and one saliva sample per time point was collected. Serum was obtained from coagulated blood samples after centrifugation for $10 \mathrm{~min}$ at $15,000 \times \mathrm{g}$ and $4^{\circ} \mathrm{C}$. Whole blood samples were briefly vortexed and centrifuged before weak sonication (Amplitude 5, 2-3 pulses; Soniprep 150 plus, MSE, London, UK) to lyse the cells. Lysates and
$0.5 \mathrm{ml}$ aliquots of the saliva samples were centrifuged for 10 min at $15,000 \times g$ and $4^{\circ} \mathrm{C}$. Supernatants were collected and immediately used for Bradford assay (see below). The remaining sample was stored at $-20^{\circ} \mathrm{C}$ until assayed for hormone content.

\section{Samples for $24 \mathrm{~h}$ hormone profiling}

Six volunteers (four females, two males, mean age \pm SD: $32 \pm 6$ years) under informed consent provided blood and saliva samples from seven time points throughout the day, starting at $11 \mathrm{a} . \mathrm{m}$. and subsequently every $4 \mathrm{~h}$ until $11 \mathrm{a}$. $\mathrm{m}$. the next day. For collection of the 11 p.m. and 3 a.m. samples, only one dim light source was allowed to prevent melatonin suppression. Approximately $1 \mathrm{ml}$ of saliva and $40 \mu 1$ of whole blood (via finger-prick) were collected. All samples were kept at $4^{\circ} \mathrm{C}$ until the end of the sampling period and processed immediately afterwards. Sample volunteers were additionally asked to complete the Munich Chronotype Questionnaire to assess individual chronotypes before participating in the study to exclude the possibility of incorporating extreme (and rare) chronotypes [8]. In addition, all sample donors reported intake of either coffee or tea 1 day before and during the sampling period, and half of the donors consumed nicotine and/or alcohol. Blood and saliva samples were treated as described above.

\section{Dried and stored samples}

A preliminary analysis was performed using blood samples with stains dried overnight on cotton and parafilm. Results were compared with samples deposited in tubes taken simultaneously and kept at room temperature before processing. To study the stability or degradation of biomolecules, three volunteers under informed consent provided blood and saliva samples at the approximate melatonin peak time (collection in dim light), and two of these subjects also provided samples within the approximate cortisol peak time. For melatonin analysis, seven blood samples $(30 \mu \mathrm{l}$ each) and seven saliva samples (140 $\mu \mathrm{l}$ each) were collected by each subject either on parafilm (one subject) or cotton (two subjects). An additional blood and saliva sample of the same volume was collected into a $1.5 \mathrm{ml}$ reaction tube as reference. For cortisol analysis, seven blood samples $(10 \mu \mathrm{l}$ each $)$ and seven saliva samples $(80 \mu \mathrm{l}$ each) were collected by each volunteer either on parafilm or cotton (one subject each), with reference samples collected in reaction tubes. Samples were kept at room temperature for $0,1,4,7,14,21$ and 28 days before storing at $-80^{\circ} \mathrm{C}$ until processing. Tube samples were frozen together with the dried T0 (time-point 0 ) samples. One cotton sample set for melatonin analysis 
was kept in the dark to control for light-dependent melatonin degradation; all other sets were kept in natural light conditions. Samples were kept on ice during preparation to reduce further degradation. Tube samples were treated as described above, with the exception that the blood tube samples were pre-diluted with ice-cold buffer for sonication due to the small sample volume. Blood and saliva stains were washed off from parafilm with an amount of buffer equal to the volume of the corresponding (prediluted) tube samples, and transferred into $1.5 \mathrm{ml}$ reaction tubes. Cotton stains were cut into small pieces and transferred into $1.5 \mathrm{ml}$ reaction tubes. The same amount of buffer was added, and samples were vortexed for $40 \mathrm{~s}$. After brief centrifugation, the supernatants were collected. To maximize the yield, the initial reaction tubes were perforated at the bottom, and placed into the tubes containing the respective supernatants. The piggyback tubes were then placed into $50 \mathrm{ml}$ reaction tubes and centrifuged for $10 \mathrm{~min}$ at $2,300 \times \mathrm{g}$ and $4^{\circ} \mathrm{C}$. All samples were assayed as described above, with the same final sample dilutions as in the 24-h profiling.

\section{Hormone assays}

The commercially available ELISA kits employed in this study are designed to measure either serum/plasma or saliva/diluted serum, with the difference that the latter kits are far more sensitive (analytical sensitivity according to manufacturer: melatonin: $1.6 \mathrm{pg} / \mathrm{ml}$ for serum assay versus $0.3 \mathrm{pg} / \mathrm{ml}$ for saliva assay; cortisol: $2.5 \mathrm{ng} / \mathrm{ml}$ for serum assay versus $0.3 \mathrm{ng} / \mathrm{ml}$ for saliva assay; for more information on precision and accuracy, please refer to the respective manuals [http://www.ibl-international.com]).

\section{Cortisol assay}

Two different cortisol ELISA kits were employed: one designed for the determination of cortisol in human saliva and diluted serum (Cortisol ELISA, RE 52611, IBL, Hamburg, Germany) and the other one designed for the determination of cortisol in human serum and plasma (Cortisol ELISA, RE 52061, IBL, Hamburg, Germany). Both assays were performed according to the manufacturer's instructions. To test the comparability and sensitivity of the assays, and to determine the optimal working conditions, serum and whole blood samples were measured with both ELISA kits in different dilutions, whereas saliva samples were only tested with the saliva kit in different dilutions. All dilutions were prepared with the zerostandard supplied with the respective kit. All samples, standards, and controls were assayed in duplicate and measured at $450 \mathrm{~nm}$ in a plate reader (Varioskan Flash, Thermo Scientific, Breda, the Netherlands).
Melatonin assay

Melatonin content in whole blood, serum, and saliva samples was determined with a commercially available ELISA kit (Non-Extraction Melatonin Saliva ELISA, IBL, Hamburg, Germany). We refrained from testing the serum kit (Melatonin ELISA, IBL, Hamburg) due to the large sample volume needed for this assay. Serum and blood samples $\left(70 \mu \mathrm{l}\right.$ each) were diluted in $\mathrm{ddH}_{2} \mathrm{O}$ (double-distilled water, to a final volume of $450 \mu \mathrm{l}$ ) and subjected to $\mathrm{C} 18$ reversed phase columns (IBL, Hamburg, Germany) according to the manufacturer's instructions for melatonin extraction. Dried extracts were reconstituted in $\mathrm{ddH}_{2} \mathrm{O}$ and assayed immediately in different dilutions per sample. All dilutions were prepared with the zero-standard supplied with the kit. All samples, standards, and controls were assayed in duplicate and according to the manufacturer's instructions. Measurement wavelength was $450 \mathrm{~nm}$ (Varioskan Flash, Thermo Scientific, Breda, the Netherlands).

Bradford assay for total protein

Total protein concentrations of serum, blood, and saliva samples were determined using a commercially available kit with bovine serum albumin as a standard (Coomassie Protein Assay Kit, Pierce, Etten-Leur, the Netherlands) in a microplate format, according to the manufacturer's instructions. In order to be within the working range of the standard protocol, $1 \mu \mathrm{l}$ of blood and $5 \mu$ of saliva samples were diluted in $199 \mu \mathrm{l}$ and $5 \mu \mathrm{l}$ ammonium acetate buffer (0.1 M, pH 6.8), respectively, containing protease and phosphatase inhibitors (Halt ${ }^{\mathrm{TM}}$ Protease and Phosphatase Inhibitor Cocktail, Pierce, Etten-Leur, Netherlands), further on just referred to as 'buffer'. Standards were prepared with the same buffer; all samples and standards were measured in duplicate $(5 \mu l$ per well) at $595 \mathrm{~nm}$ (Varioskan Flash, Thermo Scientific, Breda, Netherlands).

Statistical data analyses

Melatonin profiles were subjected to single-cosinor analysis with a 24-h period for determination of rhythmicity [9]. All other statistical analyses were performed with GraphPad Prism 5 (GraphPad Software Inc., La Jolla, USA)

\section{Results}

ELISA applicability testing

First, samples (serum, blood and saliva) were tested with a commercially available ELISA designed for determination of cortisol in human saliva and diluted serum 
samples. Optimal dilutions for serum and blood were found to be 1:50, confirming the manufacturer's information on assay sensitivity for serum. For saliva, a 1:1 dilution was chosen.

Second, the same serum and blood samples were subjected to a commercially available ELISA designed for determination of cortisol in human serum and plasma samples. Values obtained for serum and blood from the two different ELISAs were strongly correlated $(p<0.0001$, $R^{2}=0.9754$; Electronic supplementary material, Fig. S1), supporting the validity of our approach. Furthermore, due to the higher sensitivity of the saliva assay, only $1 \mu$ l instead of $20 \mu \mathrm{l}$ of sample per well were needed to reliably perform the assay. On average, cortisol concentrations obtained from blood reached $67 \% \pm 6 \%$ (mean \pm SD) of the corresponding serum concentrations using the 1:50 dilution.

Third, all three sample types were subjected to a commercially available ELISA designed for determination of melatonin in human saliva. Optimal dilutions were determined as 1:10 for blood, and 1:1 for saliva. On average, melatonin concentrations obtained from blood reached $89 \% \pm 3 \%$ (mean $\pm \mathrm{SD}$ ) of the corresponding serum concentrations using the 1:10 dilution. Corresponding values of the same individual and time point for saliva and whole blood were significantly correlated for both melatonin and cortisol $\left(p=0.0001, R^{2}=0.96\right.$ and $p=0.0002$, $R^{2}=0.95$, respectively; see Electronic supplementary material, Fig. S2).

All further experiments were conducted using the optimal working dilutions as stated above for both melatonin and cortisol from saliva and whole blood samples; for cortisol only the saliva ELISA was used in the following because of its higher sensitivity.

\section{Blood analyses}

Total protein concentration in the 24-h serial blood samples was measured to determine its potential use as a normalization parameter in the circadian hormone analyses. Remarkably stable total protein concentrations were obtained both within and between subjects (Fig. 1), with the mean value centred on $200 \mathrm{mg} / \mathrm{ml}$. There were no statistically significant inter-individual differences (oneway ANOVA: $p=0.2$ ). From measuring hormone content in the 24-h serial blood samples it became evident that all subjects displayed a typical circadian cortisol profile, with the characteristic concentration peak upon wakening ([10], see 'Reference' in Fig. 2a), and also a typical circadian melatonin profile with the characteristic concentration peak during late night-time ([10], see 'Reference' in Fig. 3a). These general patterns remained unchanged upon normalization against total protein concentration (Figs. $2 b$ and $3 b$ ).
In addition, all subjects displayed significant rhythms in their respective melatonin profiles after cosine fitting.

A preliminary analysis of blood samples dried overnight on porous (i.e. cotton) and non-porous (i.e. parafilm) surfaces was performed and results were compared with those from fresh samples taken simultaneously to obtain information on the transferability of established protocols. Total protein recovery from dried blood showed no significant concentration difference to that obtained from fresh blood (mean fresh blood: $216 \pm 56 \mathrm{mg} / \mathrm{ml}$; mean dried blood: $200 \pm 42 \mathrm{mg} / \mathrm{ml}$ ). The same was found for cortisol (mean fresh blood: $98.0 \pm 0.3 \mathrm{ng} / \mathrm{ml}$; mean dried blood: $101.5 \pm 8.4 \mathrm{ng} / \mathrm{ml}$ ) and melatonin (mean fresh blood: $39.0 \pm$ $5.8 \mathrm{pg} / \mathrm{ml}$; mean dried blood: $25.1 \pm 14.5 \mathrm{pg} / \mathrm{ml}$ ).

For time-wise stability testing, blood stains on cotton and parafilm of three individuals were kept at room temperature for up to 28 days. Total protein concentrations from aged blood samples of most individuals showed little variation over the entire sample storage period (Fig. 4). Only one individual sample set showed a statistically significant decline with increased storage time (D2 in Fig. 4), which caused a slight declining trend of the mean signal. There were no statistically significant differences between subjects (one-way ANOVA: $p=0.17$ ). Therefore, total protein concentration can be used for normalization of hormone signals in both fresh samples and aged trace samples. Determination of cortisol (Fig. 5a) and melatonin content (Fig. 5b) was possible for all dried blood samples and time points. Correlation of absolute against normalized hormone concentrations was highly significant for both cortisol and melatonin (cortisol: Pearson's $r=0.8238, p=$ 0.0003; melatonin: Pearson's $r=0.7564, p=0.0001$ ). Cortisol values obtained from both parafilm and cotton blood stains at T0 (freshly dried) were almost identical to those obtained from the respective reference tube samples of freshly frozen blood $(102 \mathrm{ng} / \mathrm{ml}$ versus $101 \mathrm{ng} / \mathrm{ml}$, and

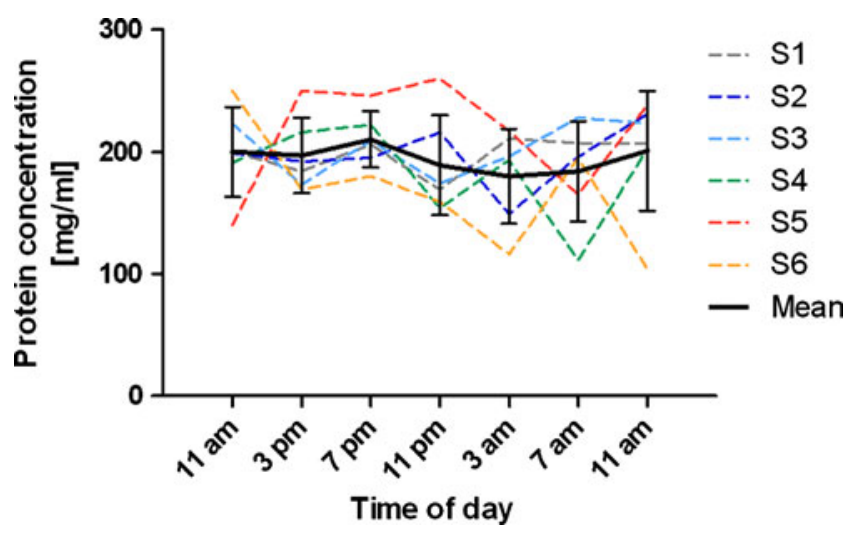

Fig. 1 Twenty-four-hour profiles of total protein concentrations in blood samples from six individuals and the respective mean $( \pm \mathrm{SD})$ values 

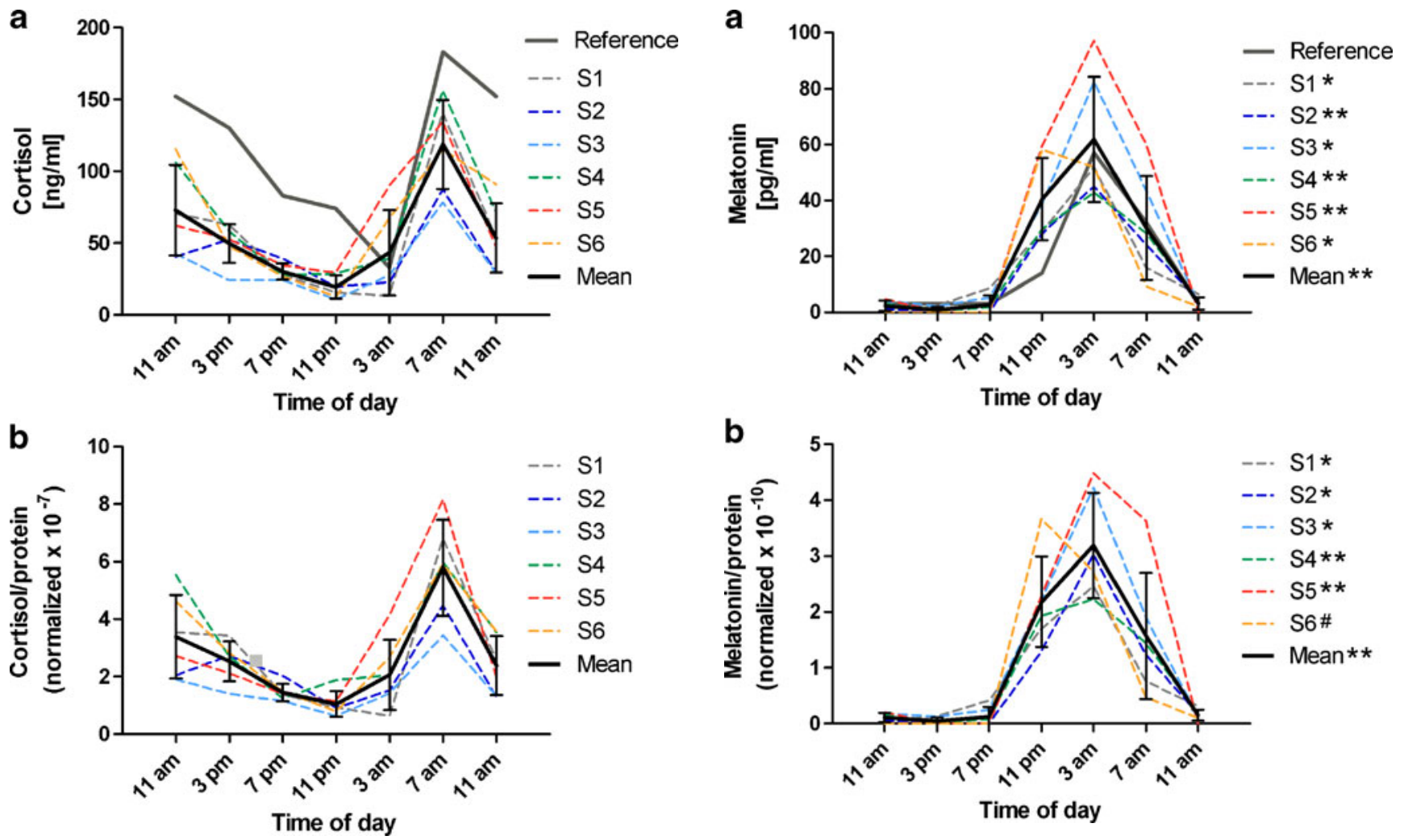

Fig. 2 Twenty-four-hour profiles of cortisol concentrations in blood samples from six individuals and the respective mean $( \pm \mathrm{SD})$ values; a reference curve (dark-gray solid line) shows the mean cortisol values obtained from serum data described elsewhere [10]

$64 \mathrm{ng} / \mathrm{ml}$ versus $68 \mathrm{ng} / \mathrm{ml}$ ). However, both of the sample sets analysed showed a statistically significant decline of the cortisol signal with time, which was retained after normalization (Fig. 5a). However, melatonin signals obtained from cotton blood samples at $\mathrm{T} 0$ were lower than those obtained from parafilm (parafilm: $81 \%$ of reference tube; cotton: mean $\pm \mathrm{SD}: 58 \% \pm 7 \%$ of reference tube), although values from the reference tube samples of freshly frozen blood were similar between samples (mean $\pm \mathrm{SD}$ : $53 \mathrm{pg} / \mathrm{ml} \pm 11 \mathrm{pg} / \mathrm{ml}$ ). Only one sample set showed a significant decrease of melatonin with storage time (D2: $p=0.04$ ), whereas the remaining two sample sets did not $(p>0.1$; data not shown). After normalization of the melatonin values, none of the sample sets showed a statistically significant decline with sample deposition time (Fig. 5b), and similar values were obtained for both cotton sample sets independent of the light condition (see D2 and D3, Fig. 5b).

Saliva analyses

Although in fresh saliva samples total protein concentration was lower and more variable during the $24-\mathrm{h}$ period compared with blood samples, with highly significant

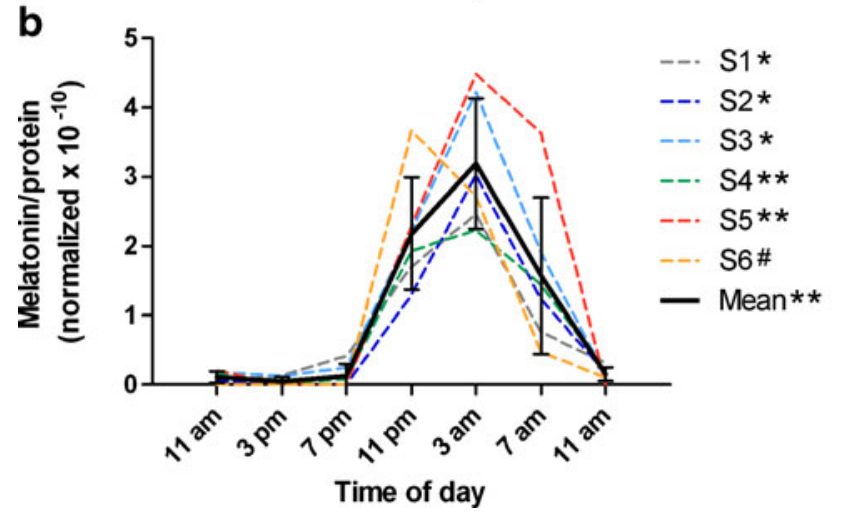

Fig. 3 Twenty-four-hour profiles of melatonin concentrations in blood samples from six subjects and the respective mean $( \pm \mathrm{SD})$ values; a reference curve (dark-gray solid line) shows the mean melatonin values obtained from serum data described elsewhere [10]; statistically significant circadian rhythms are indicated $(\# p<0.1$, ${ }^{*} p<0.05,{ }^{* *} p<0.01$ )

inter-individual differences $(p=0.0006$; Electronic supplementary material, Fig. S3), the characteristic circadian profiles could be generated for both cortisol and melatonin (Electronic supplementary materials, Figs. S4 and S5). The high saliva viscosity may have led to a higher background noise in the melatonin assay (see S2 in Electronic

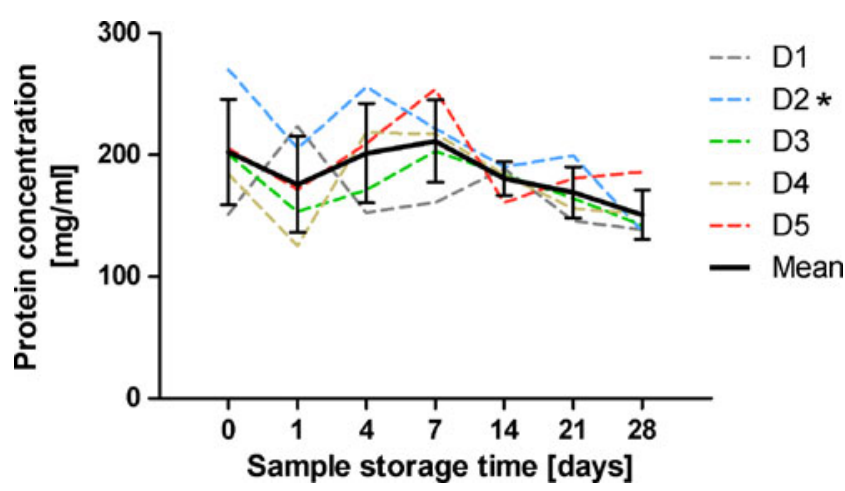

Fig. 4 Total protein concentrations obtained from five sets of dried blood stains in six time intervals over a period of 4 weeks, and the respective mean $( \pm \mathrm{SD})$ values; statistically significant decay of individual concentration values are indicated with $* p<0.05$; parafilm samples: D1 and D4, cotton samples: D2, D3, and D5 

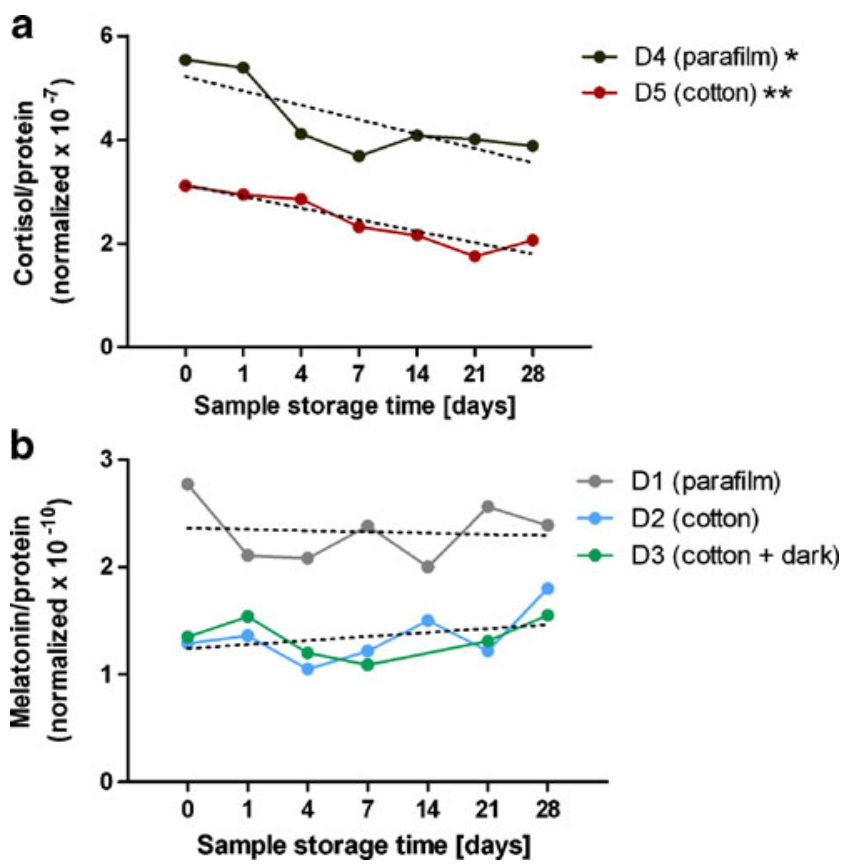

Fig. 5 a Normalized cortisol concentrations obtained from two individual sets of dried blood stains over a period of 4 weeks including the respective linear regression lines fitting the data points; statistically significant decay of individual concentration values are indicated with $* p<0.05,{ }^{*} p=0.002$; b normalized melatonin concentrations obtained from three individual sets of dried blood stains over a period of 4 weeks, including the respective linear regression lines fitting the data points for set D1 and sets D2/D3; statistically significant decay is indicated with $* p<0.05$

supplementary material, Fig. S5a). Due to the 24$\mathrm{h}$ variation in total protein concentration, normalization of hormone patterns was less successful with saliva compared to blood, which led to an occasional disappearance of the typical night time melatonin peak in the normalized analysis (see S1 in Electronic supplementary material, Fig. S5b).

Determination of total protein concentration from dried saliva stains revealed highly divergent results (data not shown). Two of the sample sets showed a significant decline of melatonin concentration with sample storage time (D1: $p=0.05, \mathrm{D} 3: p<0.0001)$, which was not observed for the third set (Electronic supplementary material, Fig. S6a). For cortisol, one of the two sets analysed showed a significant decline of cortisol concentration with sample storage time (D4: $p=0.0012$ ), whereas the other did not (Electronic supplementary material, Fig. S6b).

\section{Discussion}

With this study, we demonstrate for the first time the feasibility of estimating deposition time of biological traces by means of circadian biomarkers. Our approach includes determination of melatonin and cortisol concentrations and their normalization against total protein concentration to control for variation in sample input. This biochemical tool is expected to enable a forensic researcher to narrow down the time of deposition of a trace sample collected at a crime scene to either late night (melatonin) or early morning (cortisol). We chose a simple method based on ELISA because (1) it allows the use of commercially available assays that are fully established in clinical diagnostics including quality control, (2) sample processing is straightforward and (3) only basic laboratory equipment is needed. Alternatives for hormone quantifications include chromatographic methods such as GC/MS or LC/MS. Studies of human circadian rhythms aiming to establish biological relationships normally involve strictly controlled laboratory conditions (i.e. control of lighting levels, food intake, posture, sleep etc.) for sampling, including also the socalled constant routine protocol, which is used to unmask endogenous rhythms from any confounding factors $[4,11$, 12]. However, such controlled conditions were not applied in our study aiming to provide a tool for future forensic applications, and it is highly remarkable that we were still able to reproduce both hormone profiles with high accuracy. This provides encouraging evidence that our approach can be transferred to forensic practice.

One key aspect of the ELISA assay applicability testing was downscaling of the sample volumes necessary for the assays to meet requirements of forensic trace analysis. With the almost perfect correlation of the values obtained for the same blood and serum samples using two different cortisol ELISA kits, we were able to achieve this goal with only $1 \mu \mathrm{l}$ of sample (instead of $20 \mu \mathrm{l}$ ) needed. In addition, in order to use whole blood samples with the melatonin saliva kit employed in this study, melatonin was extracted from the samples to prevent matrix problems and false measurements before applying the samples to the ELISA plate. This extraction procedure has already been proven as feasible approach using homogenized tissue of post-mortem human pineal glands [6]. Overall, a total volume of $25 \mu \mathrm{l}$ of blood, corresponding to a cotton blood stain size of only $70 \mathrm{~mm}^{2}$, is required to perform all melatonin, cortisol and total protein measurements in duplicate. A minimal cotton blood sample size of $50 \mathrm{~mm}^{2}$ would be required if single measurements were employed. In contrast, a much higher total volume of $160 \mu \mathrm{l}$ saliva was needed for all measurements in duplicates.

The highly significant correlations between corresponding values for saliva and blood for both melatonin and cortisol are in agreement with previously published data comparing hormone concentrations between blood, serum, and saliva [13-16]. The observation that cortisol concentrations obtained from whole blood reached only $67 \%$ on average when compared with the corresponding serum concentra- 
tions may be explained by the dilution of the serum component with the rest of the blood compounds present in whole blood, leaving the same amount of cortisol in a larger volume for the assay. However, this effect was not seen with melatonin, where similar values from blood and serum were obtained. It has been shown recently that melatonin is also produced by immune cells present in the blood, providing a melatonin pool that may not be accessible in serum alone [17].

The $24 \mathrm{~h}$ profiling for total protein concentration and hormone levels could be successfully performed for blood samples. The finding of a remarkably stable total protein concentration during the entire day-night period, both within and between subjects, is in agreement with previous data on serum and cellular blood components [18]. Hence, these data allow the conclusion that normalization of hormone levels against total protein levels can be considered a valid approach. Both melatonin and cortisol profiles showed the typical $24 \mathrm{~h}$ pattern, with subjects who were employed in different parts of this study (validation, profiling, degradation) exhibiting high reproducibility [4, 10-12, 19]. Comparison of our calculated mean hormone patterns with the respective reference curves also confirmed the previous statement that melatonin values in blood reach similar levels to those in serum, whereas values for cortisol are found to be lower [10].

Our preliminary blood stain analysis revealed a good transferability of the methods established on fresh blood. Surprisingly, the recovery rate of total protein and cortisol came very close to the reference tubes from freshly frozen samples, independent of the surface material. For melatonin, the recovery rate was lower (although not statistically significant). As expected, resolubilisation of dried blood was achieved more easily from non-porous surfaces. When testing blood samples stored up to 4 weeks, total protein concentrations were in general surprisingly stable across the entire time period within and between individuals. As such, we conclude that the use of this parameter for normalization is a valid approach for forensic samples, at least within the storage time limit of 4 weeks tested in our study. This conclusion was strengthened by the highly significant correlations found for measured against normalized values for both hormones. In our hands, melatonin concentrations from blood samples did not decrease significantly with sample storage time of 4 weeks after normalization, which is in line with previous data on post-mortem melatonin degradation [7]. Interestingly, there was no remarkable difference observed between the sample set kept in the dark and the one kept near the window, suggesting that, at least under these experimental conditions, photodegradation of melatonin is negligible [20]. Although there is no report on melatonin stability in stains thus far, it should be mentioned here that melatonin is reported to be photolabile in solution
[20]. On the other hand, cortisol levels from blood declined significantly with sample storage time, which is in contrast to previous reports on the stability of cortisol in serum and whole blood, yet to our knowledge there is no study on the stability of cortisol in blood stains on cotton or parafilm in the literature [21, 22]. Therefore, the observed time-wise decay suggests that the use of this hormone may be difficult in samples stored for longer time intervals. We would advise the implementation of a strict threshold value for use and interpretation of the result. In our albeit limited study, the cotton sample set would be used as a reference and lead to a threshold value of $2.5 \times 10^{-7}$ (cortisol/protein normalized), or 7 days degradation time, below which no more conclusions can be drawn on the sample deposition time. However, we would like to emphasize that more detailed forensic validation studies are needed to establish more stringent regulations for this issue.

One interesting question raised by our study is whether the same stain sample can be used for multiple purposes. To test this, we used part of the melatonin extraction column flow-through from two $20 \mu \mathrm{l}$ fresh blood samples for DNA extractions applying the QIAamp DNA Micro extraction system (Qiagen), and were able to generate complete DNA profiles with the AmpFlSTR Identifiler PCR amplification kit (Applied Biosystems). In addition, by using another part of the same flow-through it was possible to extract total RNA after phenol extraction with the RNeasy Micro Kit (Qiagen), to synthesise cDNA with the RevertAid H Minus First Strand cDNA Synthesis Kit (Fermentas), and to successfully perform mRNA profiling for blood specific markers (data not shown) [23].

In general, the situation in saliva turned out to be more difficult compared to whole blood, providing less consistent and firm results. The large differences observed in total protein concentrations within and between individuals confirm previous findings, and do not allow recommending this parameter for normalization [18, 24]. Also, the amount of saliva needed for the assays might affect saliva usability in forensic trace analysis. High-viscosity saliva resulted in falsely increased melatonin values, and for some of the subjects the peak in total protein concentration fell together with the melatonin peak in the middle of the night, cancelling out each other in normalization. It has been described before that protein degradation in saliva is extremely rapid compared to other biological fluids, and starts immediately upon sample collection [25]. The dynamic nature of the saliva proteome shows high variation in protein concentration between and within individuals [18, 24]. Thus, it remains to be shown if highly stable protein markers with constant $24 \mathrm{~h}$ expression can be established. Until such markers for normalization of variation in saliva input are identified, the use of saliva in forensic applications of circadian biomarkers is discouraged. 
It shall be mentioned that the use of the circadian hormones melatonin and cortisol in future forensic applications is not without limitations. One aspect is that some individuals represent extreme chronotypes ('owls' or 'larks'; approximately $2 \%$ of the population, not part of our study) with significantly shifted profiles in their circadian rhythms. Furthermore, night-time melatonin biosynthesis is suppressible by exposure to light $[3,26,27]$. It has been shown that a three hour night-time light exposure (600 lux) decreased melatonin concentrations by $65 \%$, whereas cortisol levels were not significantly altered [28]. Furthermore, an association between reduced melatonin levels with depression is reported in the literature but discussed controversially [29, 30]. Cortisol, besides its role as a circadian marker, is also known as biomarker for stress. Although it is not immediately up-regulated in acute (psychological) stress situations but instead exhibits a delayed response, implying little or no influence on crime scene samples [31, 32]. Yet, several human and animal studies suggest associations between altered cortisol levels and physical or mental symptoms, like posttraumatic stress disorder or addiction (see [5,33] and references therein). Another aspect is that our approach is based on the fact that every human being is entrained to the environment, mainly by the synchronizing factor light. Yet there are a few exceptions from this general principle, like blind persons who have no conscious light perception and therefore may show shifted circadian patterns [34]. Disturbed circadian rhythms can also be found for some days after transmeridian flights ('jet-lag') and in shift workers with slowly rotating schedules (see [35], and references therein). Furthermore, reduced amplitudes in circadian rhythms, including the melatonin rhythm, have been described in the elderly, although this phenomenon needs to be studied more systematically $[36,37]$. Thus, data obtained for both melatonin and cortisol in future forensic applications need to be interpreted carefully and in the context of available information on the case background.

Despite of the potential caveats listed we believe that the presented approach based on the circadian hormones melatonin and cortisol bears large potentials for a first estimation of the time a biological trace was deposited at a crime scene, which can provide crucial information for solving crimes. Systematic studies in search for more circadian biomarkers shall be carried out in the future to see if the time range offered by the two hormones studied here can be specified more exactly. In addition, our approach may be transferable to forensic cases involving whole bodies rather than traces: Pineal melatonin content has been suggested as an additional indicator for estimating time of death [7], yet confounding factors such as depression/suicide have to be considered [38]. Also, melatonin levels in different body sites were found to be not significantly related to post-mortem interval or gender, but highly correlated with each other [16]. Still, future research is needed to confirm the feasibility of such an approach. Besides the anticipated forensic application, there may also be clinical applications for the described methods. The small blood volume needed to perform the circadian hormone analyses could be beneficial, for instance in the monitoring of disturbed melatonin production and/or its supplementation treatment in premature infants, or taking advantage of the easily performed sampling via fingerprick, which allows for multiple sampling even in children or in elderly people in a domestic environment, a situation often found in field studies on ageing or biological rhythm disorders [39-42].

Acknowledgements The authors thank all the volunteers contributing to this study. Mijke Visser is thanked for performing RNA extractions and mRNA profiling for blood identification. We thank Prof. Debra J. Skene for helpful comments on the manuscript as well as the anonymous reviewers for constructive criticism.

This work was supported by the Netherlands Forensic Institute (NFI), and received additional support by a grant from the Netherlands Genomics Initiative (NGI)/Netherlands Organization for Scientific Research (NWO) within the framework of the Forensic Genomics Consortium Netherlands (FGCN).

Conflict of Interest The authors declare that they have no conflict of interest.

Open Access This article is distributed under the terms of the Creative Commons Attribution Noncommercial License which permits any noncommercial use, distribution, and reproduction in any medium, provided the original author(s) and source are credited.

\section{References}

1. Anderson S, Howard B, Hobbs GR, Bishop CP (2005) A method for determining the age of a bloodstain. Forensic Sci Int 148:37-45

2. Hastings M (1998) The brain, circadian rhythms, and clock genes. Bmj 317:1704-1707

3. Arendt J (2006) Melatonin and human rhythms. Chronobiol Int 23:21-37

4. Viola AU, Archer SN, James LM, Groeger JA, Lo JC, Skene DJ, von Schantz M, Dijk DJ (2007) PER3 polymorphism predicts sleep structure and waking performance. Curr Biol 17:613-618

5. Fries E, Dettenborn L, Kirschbaum C (2009) The cortisol awakening response (CAR): facts and future directions. Int $\mathrm{J}$ Psychophysiol 72:67-73

6. Ackermann K, Bux R, Rub U, Korf HW, Kauert G, Stehle JH (2006) Characterization of human melatonin synthesis using autoptic pineal tissue. Endocrinology 147:3235-3242

7. Mikami H, Terazawa K, Takatori T, Tokudome S, Tsukamoto T, Haga K (1994) Estimation of time of death by quantification of melatonin in corpses. Int J Legal Med 107:42-51

8. Zavada A, Gordijn MC, Beersma DG, Daan S, Roenneberg T (2005) Comparison of the Munich chronotype questionnaire with the Horne-Ostberg's morningness-eveningness score. Chronobiol Int 22:267-278 
9. Minors D, Waterhouse J (1989) Analysis of biological time series. In: Arendt J, Minors D, Waterhouse J (eds) Biological rhythms in clinical practice. Wright, London, pp 272-293

10. Skene DJ, Bojkowski CJ, Arendt J (1994) Comparison of the effects of acute fluvoxamine and desipramine administration on melatonin and cortisol production in humans. Br J Clin Pharmacol $37: 181-186$

11. Hofstra WA, de Weerd AW (2008) How to assess circadian rhythm in humans: a review of literature. Epilepsy Behav 13:438444

12. Skene DJ, Arendt J (2006) Human circadian rhythms: physiological and therapeutic relevance of light and melatonin. Ann Clin Biochem 43:344-353

13. Dorn LD, Lucke JF, Loucks TL, Berga SL (2007) Salivary cortisol reflects serum cortisol: analysis of circadian profiles. Ann Clin Biochem 44:281-284

14. Kennaway DJ, Voultsios A (1998) Circadian rhythm of free melatonin in human plasma. J Clin Endocrinol Metab 83:10131015

15. Wong V, Yan T, Donald A, McLean M (2004) Saliva and bloodspot cortisol: novel sampling methods to assess hydrocortisone replacement therapy in hypoadrenal patients. Clin Endocrinol (Oxf) 61:131-137

16. Sturner WQ, Lynch HJ, Deng MH, Gleason RE, Wurtman RJ (1990) Melatonin concentrations in the sudden infant death syndrome. Forensic Sci Int 45:171-180

17. Carrillo-Vico A, Lardone PJ, Fernandez-Santos JM, MartinLacave I, Calvo JR, Karasek M, Guerrero JM (2005) Human lymphocyte-synthesized melatonin is involved in the regulation of the interleukin-2/interleukin-2 receptor system. J Clin Endocrinol Metab 90:992-1000

18. Crosley LK, Duthie SJ, Polley AC, Bouwman FG, Heim C, Mulholland F, Horgan G, Johnson IT, Mariman EC, Elliott RM, Daniel H, de Roos B (2009) Variation in protein levels obtained from human blood cells and biofluids for platelet, peripheral blood mononuclear cell, plasma, urine and saliva proteomics. Genes Nutr 4:95-102

19. Selmaoui B, Touitou Y (2003) Reproducibility of the circadian rhythms of serum cortisol and melatonin in healthy subjects: a study of three different 24-h cycles over six weeks. Life Sci 73:3339-3349

20. Andrisano V, Bertucci C, Battaglia A, Cavrini V (2000) Photostability of drugs: photodegradation of melatonin and its determination in commercial formulations. J Pharm Biomed Anal 23:15-23

21. Kley HK, Rick W (1984) The effect of storage and temperature on the analysis of steroids in plasma and blood. J Clin Chem Clin Biochem 22:371-378

22. Kubasik NP, Ricotta M, Hunter T, Sine HE (1982) Effect of duration and temperature of storage on serum analyte stability: examination of 14 selected radioimmunoassay procedures. Clin Chem 28:164-165

23. Zubakov D, Hanekamp E, Kokshoorn M, van Ijcken W, Kayser M (2008) Stable RNA markers for identification of blood and saliva stains revealed from whole genome expression analysis of timewise degraded samples. Int J Legal Med 122:135-142

24. Helmerhorst EJ, Oppenheim FG (2007) Saliva: a dynamic proteome. J Dent Res 86:680-693

25. Esser D, Alvarez-Llamas G, de Vries MP, Weening D, Vonk RJ, Roelofsen H (2008) Sample stability and protein composition of saliva: implications for its use as a diagnostic fluid. Biomark Insights 3:25-27

26. Bojkowski CJ, Aldhous ME, English J, Franey C, Poulton AL, Skene DJ, Arendt J (1987) Suppression of nocturnal plasma melatonin and 6-sulphatoxymelatonin by bright and dim light in man. Horm Metab Res 19:437-440

27. Lewy AJ, Wehr TA, Goodwin FK, Newsome DA, Markey SP (1980) Light suppresses melatonin secretion in humans. Science 210:1267-1269

28. McIntyre IM, Norman TR, Burrows GD, Armstrong SM (1992) Melatonin, cortisol and prolactin response to acute nocturnal light exposure in healthy volunteers. Psychoneuroendocrinology $17: 243-248$

29. Beck-Friis J, von Rosen D, Kjellman BF, Ljunggren JG, Wetterberg L (1984) Melatonin in relation to body measures, sex, age, season and the use of drugs in patients with major affective disorders and healthy subjects. Psychoneuroendocrinology 9:261-277

30. Thompson C, Franey C, Arendt J, Checkley SA (1988) A comparison of melatonin secretion in depressed patients and normal subjects. Br J Psychiatry 152:260-265

31. Noto Y, Sato T, Kudo M, Kurata K, Hirota K (2005) The relationship between salivary biomarkers and state-trait anxiety inventory score under mental arithmetic stress: a pilot study. Anesth Analg 101:1873-1876

32. Takatsuji K, Sugimoto Y, Ishizaki S, Ozaki Y, Matsuyama E, Yamaguchi Y (2008) The effects of examination stress on salivary cortisol, immunoglobulin $\mathrm{A}$, and chromogranin $\mathrm{A}$ in nursing students. Biomed Res 29:221-224

33. Lovallo WR (2006) Cortisol secretion patterns in addiction and addiction risk. Int J Psychophysiol 59:195-202

34. Hack LM, Lockley SW, Arendt J, Skene DJ (2003) The effects of low-dose 0.5 -mg melatonin on the free-running circadian rhythms of blind subjects. J Biol Rhythms 18:420-429

35. Rajaratnam SM, Arendt J (2001) Health in a 24-h society. Lancet 358:999-1005

36. Iguichi H, Kato KI, Ibayashi H (1982) Age-dependent reduction in serum melatonin concentrations in healthy human subjects. J Clin Endocrinol Metab 55:27-29

37. Skene DJ, Swaab DF (2003) Melatonin rhythmicity: effect of age and Alzheimer's disease. Exp Gerontol 38:199-206

38. Stanley M, Brown GM (1988) Melatonin levels are reduced in the pineal glands of suicide victims. Psychopharmacol Bull 24:484-488

39. Dowling GA, Burr RL, Van Someren EJ, Hubbard EM, Luxenberg JS, Mastick J, Cooper BA (2008) Melatonin and bright-light treatment for rest-activity disruption in institutionalized patients with Alzheimer's disease. J Am Geriatr Soc 56:239-246

40. Jan JE, Wasdell MB, Freeman RD, Bax M (2007) Evidence supporting the use of melatonin in short gestation infants. J Pineal Res 42:22-27

41. Riemersma-van der Lek RF, Swaab DF, Twisk J, Hol EM, Hoogendijk WJ, Someren EJ Van (2008) Effect of bright light and melatonin on cognitive and noncognitive function in elderly residents of group care facilities: a randomized controlled trial. Jama 299:2642-2655

42. Van der Heijden KB, Smits MG, Van Someren EJ, Gunning WB (2005) Idiopathic chronic sleep onset insomnia in attention-deficit/ hyperactivity disorder: a circadian rhythm sleep disorder. Chronobiol Int 22:559-570 